\title{
Design and Analysis of Rack and Pinion Mechanism in Automobile Applications Using Structural Steel and PLA
}

Shubham Torvi*, Vaishnavi Ingale, Rajkumar E.

School of Mechanical and Building Sciences, Vellore Institution of Technology, Vellore, Tamil Nadu, India

\section{ABSTRACT}

In this study, the analysis of rack and pinion used in various applications is performed. It is the most common steering linkage used in cars. Apart from cars, it is also used in many applications like press, stair lift, rack railway, actuators, etc. This study contains the analysis of rack and pinion used in automobiles and rack and pinion press. The analysis is done using traditional materials like structural steel as well as polylactic acid (PLA). PLA material is biodegradable in nature. Already existing manufacturing equipment can be used to produce it. This makes it relatively cost efficient to produce. Solidworks software is used for designing and ANSYS is used for the analysis. The results of structural steel are compared with PLA and the product is $3 D$ printed for experimentation and demonstration purpose. The innovations in the world of additive manufacturing and $3 D$ printing is opening wide area of scope to produce light-weight, high-strength and ease-in-manufacturing complex geometry as compared to traditional manufacturing techniques.

Keywords: analysis, 3D printing, design, PLA, rack and pinion, structural steel

\section{*Corresponding Author}

E-mail: shubhamtorvi007@gmail.com

\section{INTRODUCTION}

Rack and pinion adapt changes over revolution motion into linear motion. The level and toothed part is known as the rack and the gear part is known as the pinion. The rack and pinion mesh with each other in order to change the direction of wheel and converts the direction of motion. Rack and pinion is used for various activities like mechanism used for lifting objects (vertical movement), horizontal movement, positioning oriented mechanisms, stoppers, etc. They are also used in steering systems in automobiles to change the direction. Rack and pinion has the accompanying qualities: straightforward structure, high unbending nature, minimized and lightweight, and touchy and sensitive responsiveness. The pinion which is mounted to the guiding shaft is fitted with a directing rack to transmit rotating movement along the side

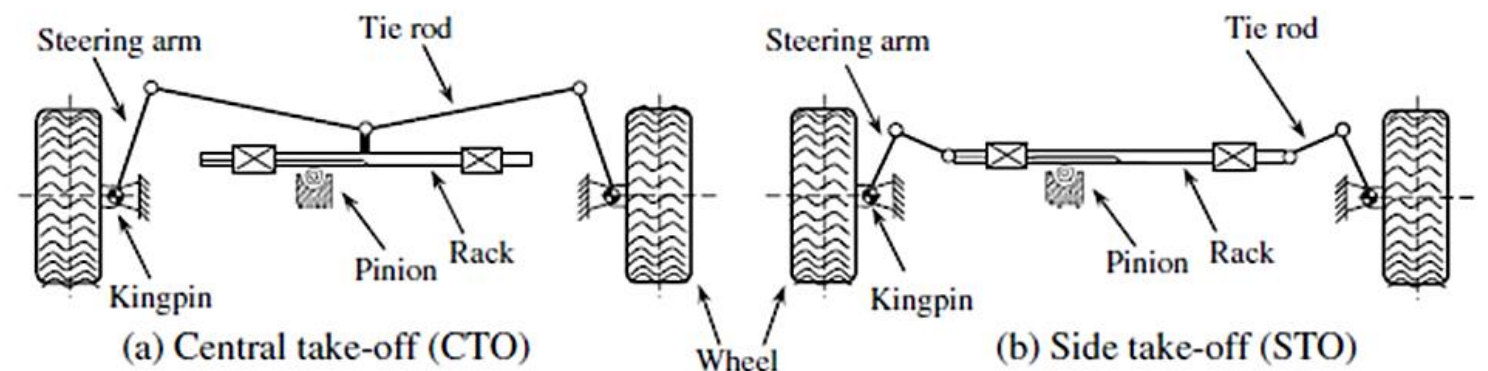

Fig. 1. Rack and pinion linkages and its configuration. 
(changing over it to straight motion) in request to control the turning of wheel. It is also used in toys, press, etc. In steering, the linkage has two common configurations, namely the central take-off (CTO) and side take-off (STO). CTO refers to the connection of rack and tie rods to the middle of rack, while STO refers to rack and tie rods connected to rack ends. Figure 1-2.

\section{LITERATURE REVIEW}

Ashwin Chopane et al. [1] carried out the design and analysis of gears in rack and pinion steering system made from plastic for formula supra car. The plastic used was Nylon 66. The study concluded that the usage of plastic gears in a supra car is very beneficial, as it aids in reducing vehicle weight and also reduction in vibrations and noise. Rahmani Hankazi et al. [2], in his study, has performed the combined kinematic and sensitivity optimization of a rack and pinion steering linkage. The kinematic optimization of the linkage is carried out by using three homogenous design parameters. One of the observation is that the sensitivities of the steering error with respect to the variations of different design parameters vary widely. Sandro Pereira dos Santos et al. [3] performed the finishing process analysis between honing and hard hobbing in pinion gears applied to a steering system. The results highlight that the honing process gives a finishing surface and roughness with high superior quality that enables engagement between surface gears without any noise [4].

\section{METHODOLOGY}

Step 1: Design a CAD model using Solidworks or any other software.

Step 2: Design the rack and pinion separately and do the assembly.

Step 3: Do the 3D static structural analysis of rack and pinion on different forces to simulate the real-time conditions and get the optimum results. First, the analysis is done on structural steel and then using poly lactic acid (PLA).

Step 4: Define the parameters and get the Ansys results.

Step 5: Compare the results and give the conclusion of both the materials.

Step 6: Simulate on Cura software and print a scaled-down 3D printed model. The methodology steps are shown in Figure 3(a).

\section{MODELLING}

The isometric view of the rack and pinion is shown in Figure 3 has geometry of rack and pinion separately with all the dimensions is also presented in Figure 4. The exploded view is given as three parts to provide easy understanding of the assembly (Figures 3-8).

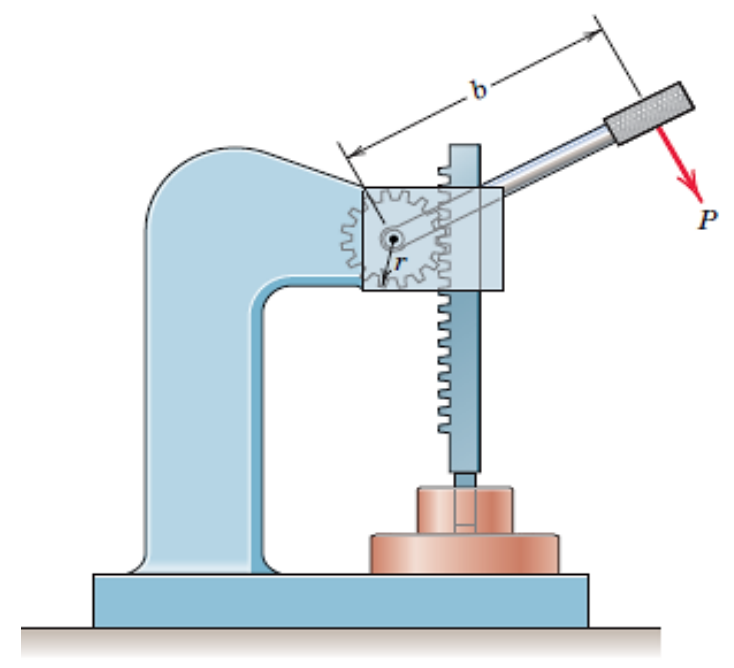

Fig. 2. Rack and pinion press. 


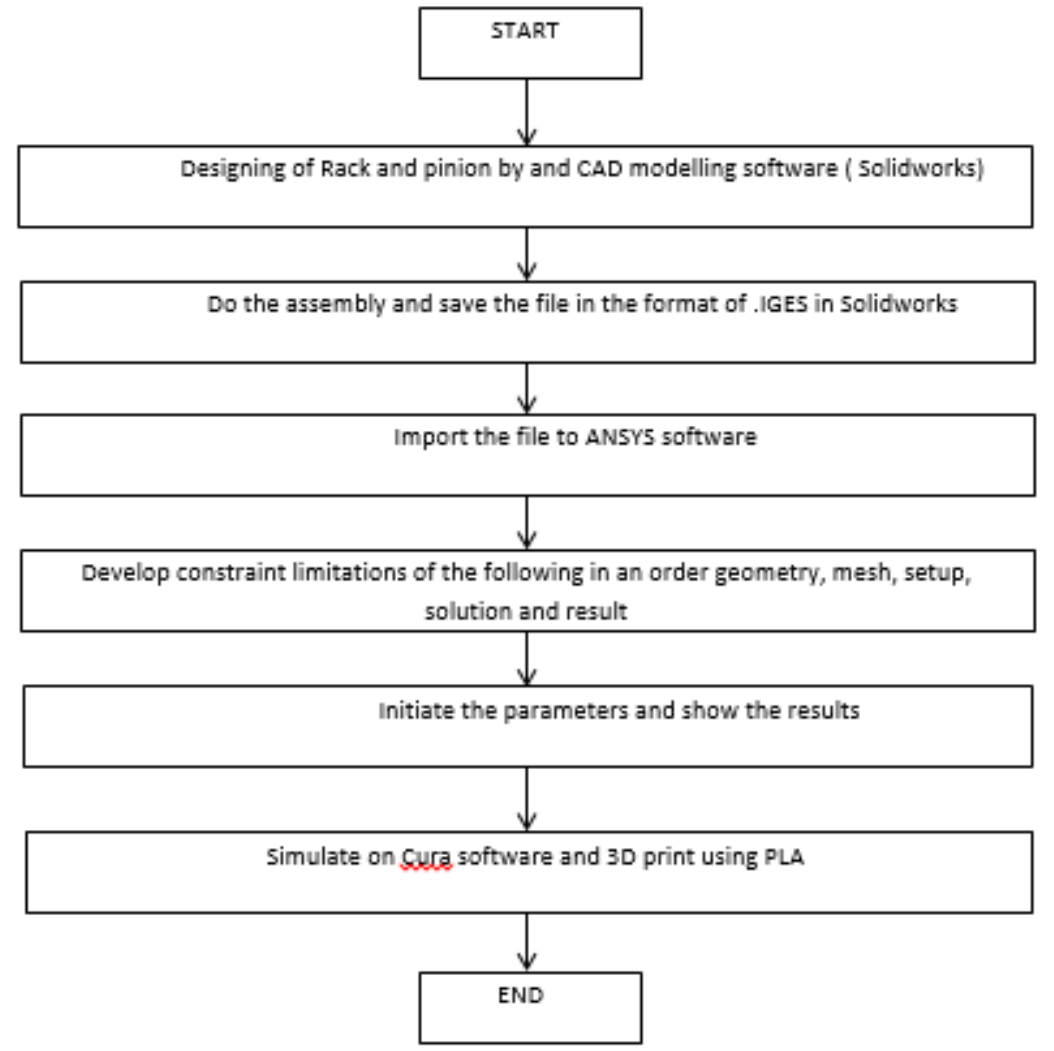

Fig. 3(a). Methodology.

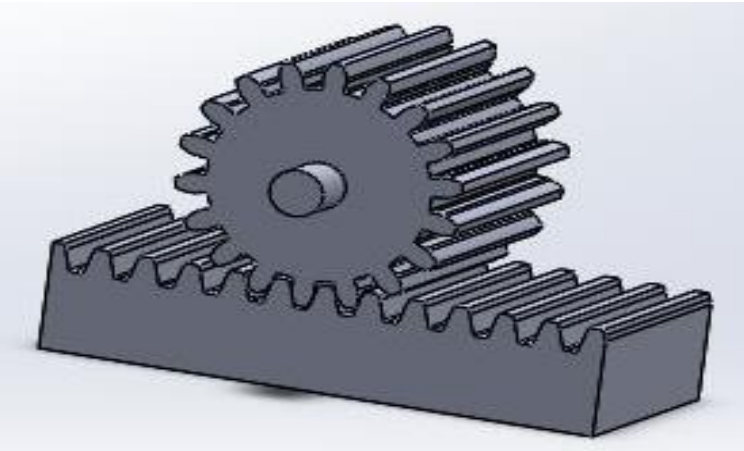

Fig. 3(b). Rack and pinion assembly.

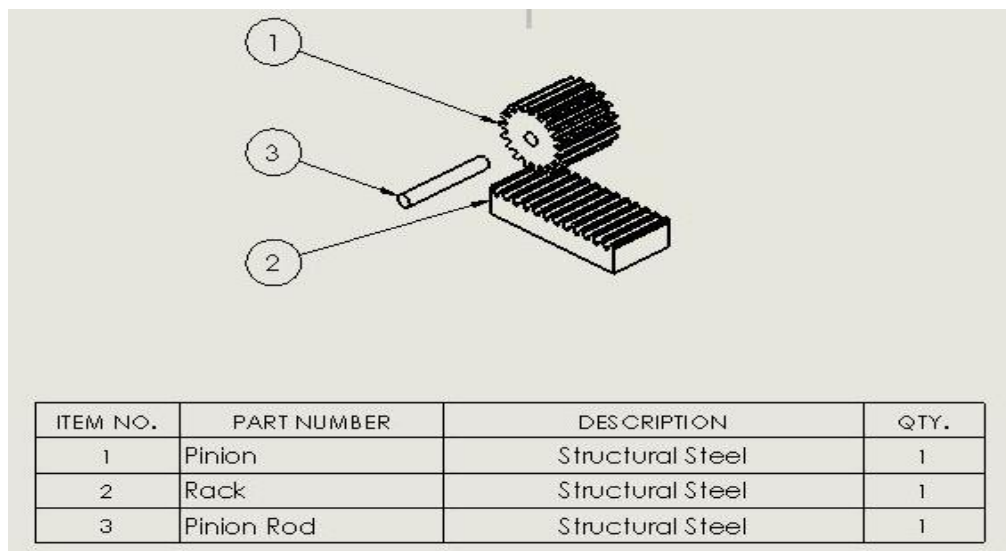

Fig. 4. Exploded view and BOM. 


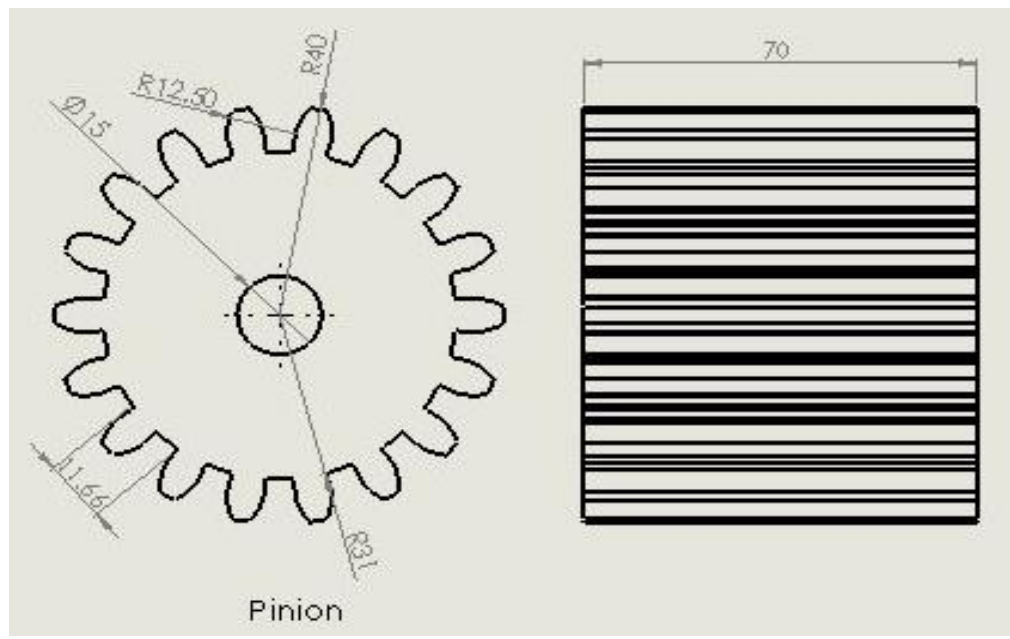

Fig. 5. Two-dimensional sketch of pinion.
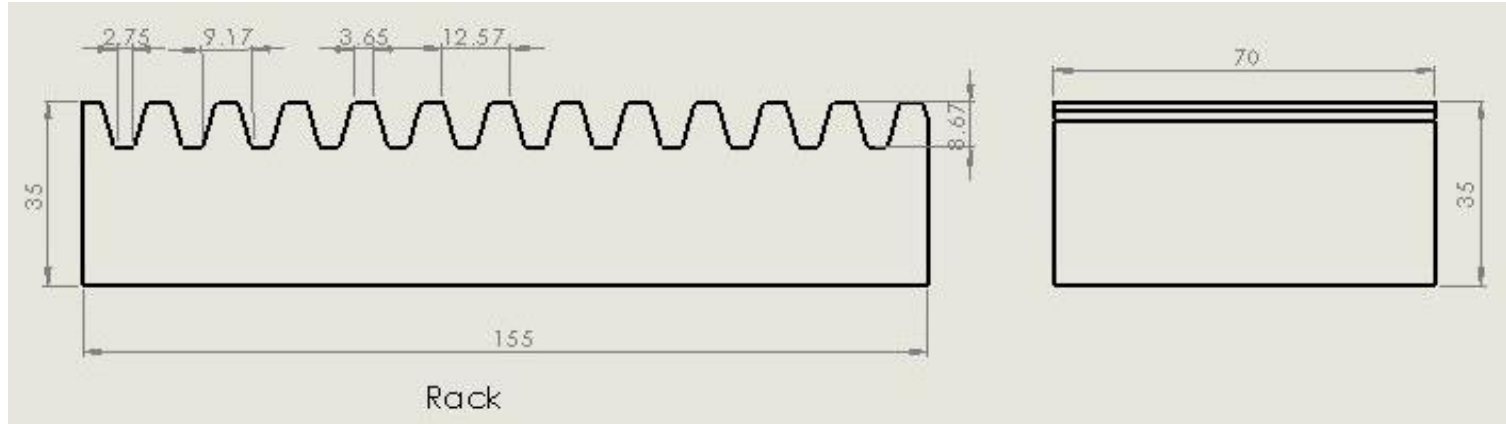

Fig. 6. Two-dimensional sketch of rack.

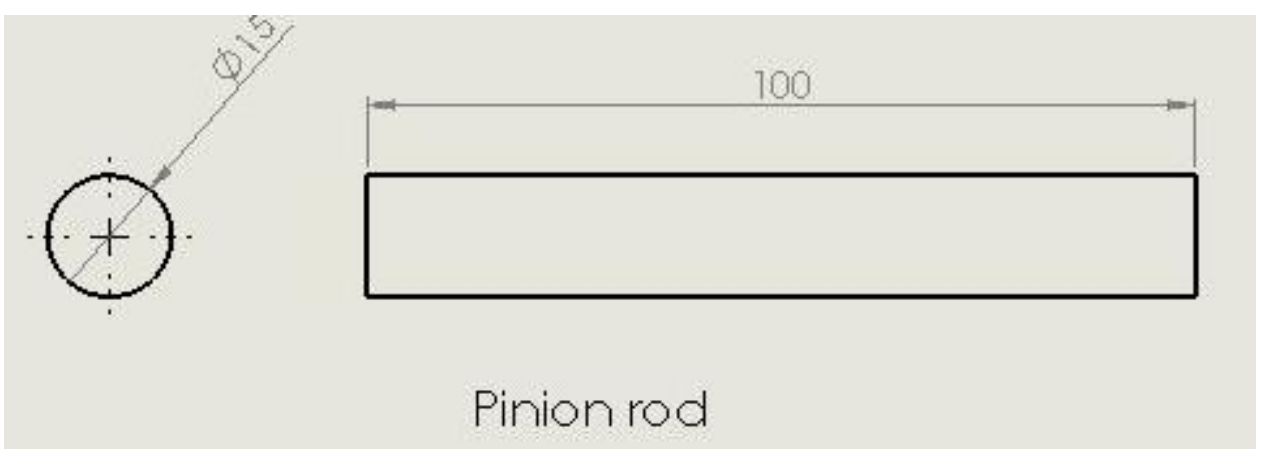

Fig. 7. Two-dimensional sketch of pinion rod.

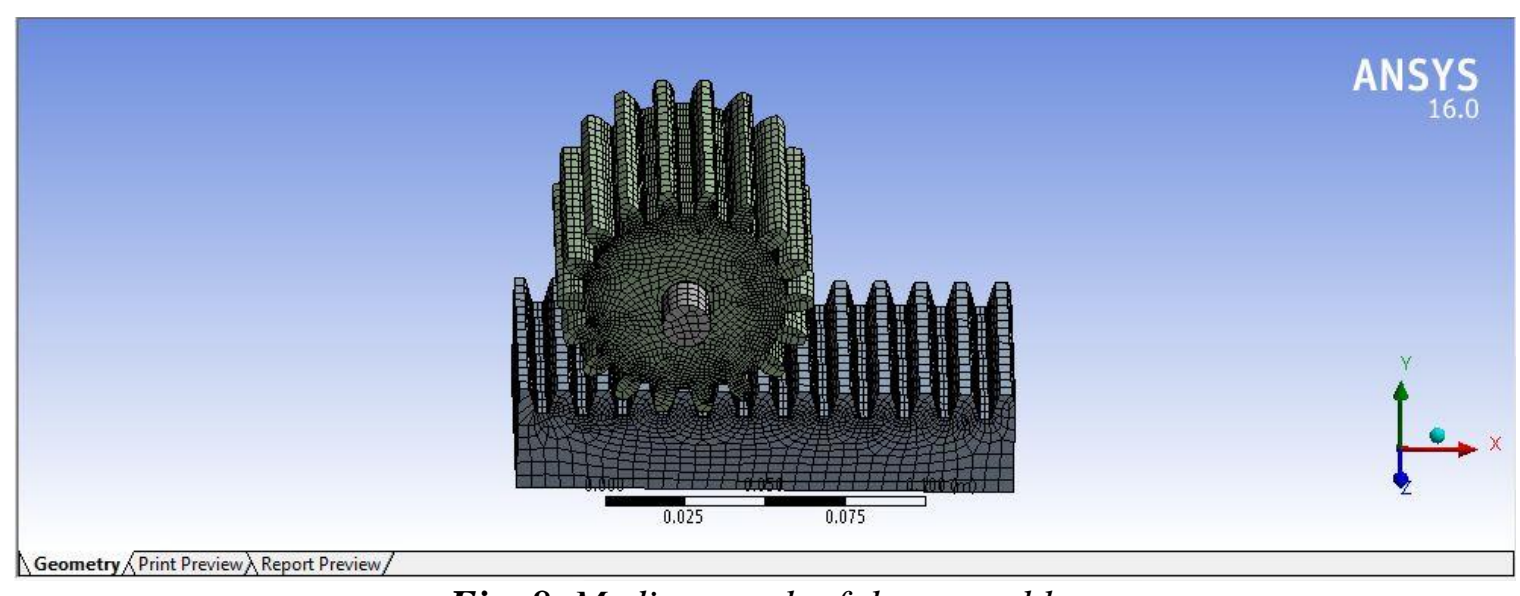

Fig. 8. Medium mesh of the assembly. 


\section{EXPERIMENTATION}

(i) The first analysis contains static structural analysis of rack and pinion with a force of $300 \mathrm{~N}$ acting in the $X$ component of the rack. The pinion rod is given relative displacement $(0,0,0)$ in all directions. The maximum force in steering is considered to be $300 \mathrm{~N}$. The mesh sizing is taken as medium. This analysis is done for rack and pinion used in automobile steering using structural steel material.

(ii) The second analysis contains static structural analysis of rack and pinion with a force of $2500 \mathrm{~N}$ acting in the $X$ component of rack. The mesh sizing is taken as medium. This analysis is done for the press which experiences a force of $2500 \mathrm{~N}$. The material selected is structural steel.

(iii)The third analysis contains static structural analysis of rack and pinion with a force of $300 \mathrm{~N}$ acting in the $X$ component of rack. The material used here is PLA, which is widely used for 3D printing. The mesh sizing is taken as medium. This analysis is done for rack and pinion used in automobile steering using PLA material.

(iv)The fourth analysis contains static structural of rack and pinion at $1000 \mathrm{~N}$ for press application using PLA material.

\section{RESULTS AND DISCUSSION}

The analysis, as shown in Figures 9 and 10 , states that the maximum deformation occurs at the rack where the force is acting upon and also on the surface contact area of the teeth of rack and pinion. The deformation is also very low with $1.8501 \times$ $10^{-5} \mathrm{~m}$ being the maximum.

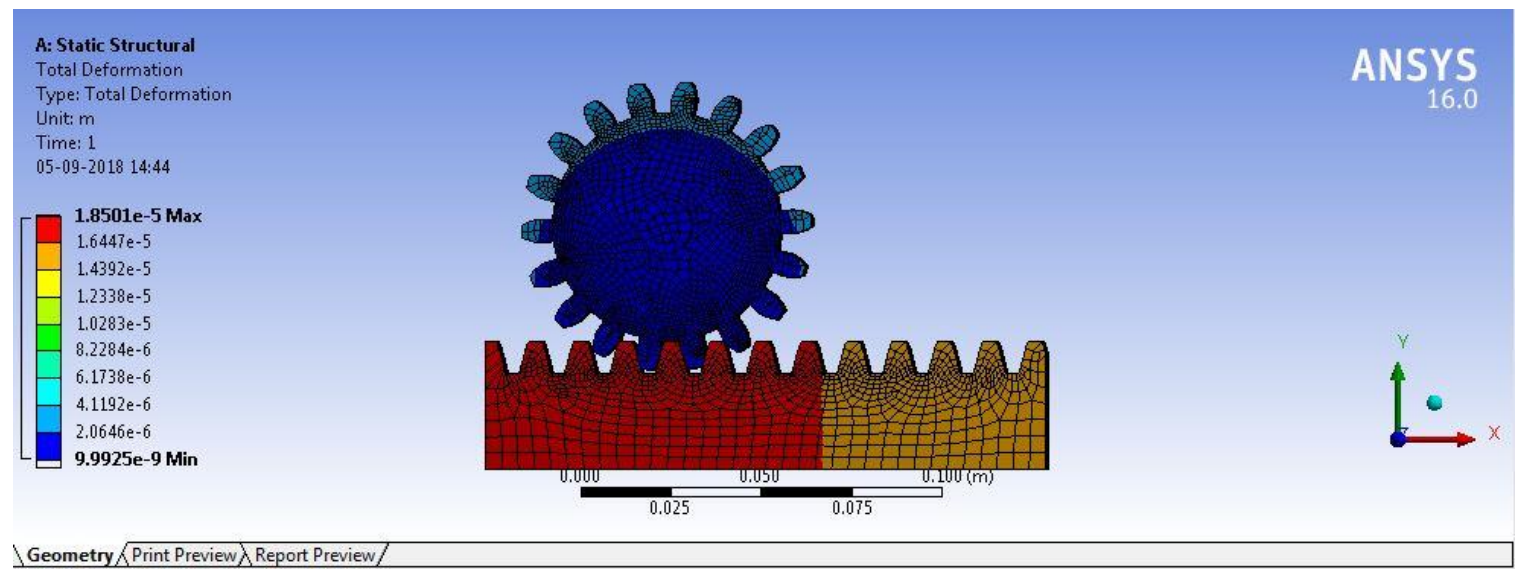

Fig. 9. Total deformation of rack and pinion at $300 \mathrm{~N}$ using SS.

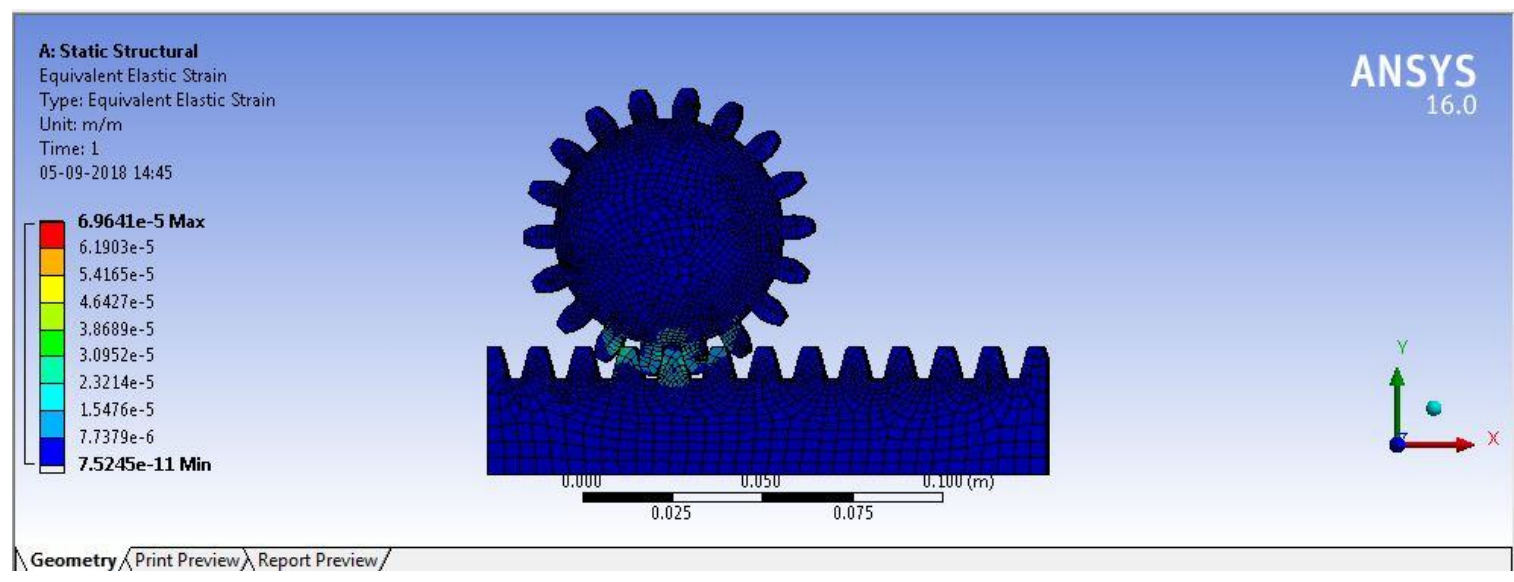

Fig. 10. Equivalent elastic strain of rack and pinion at $300 \mathrm{~N}$ using SS. 


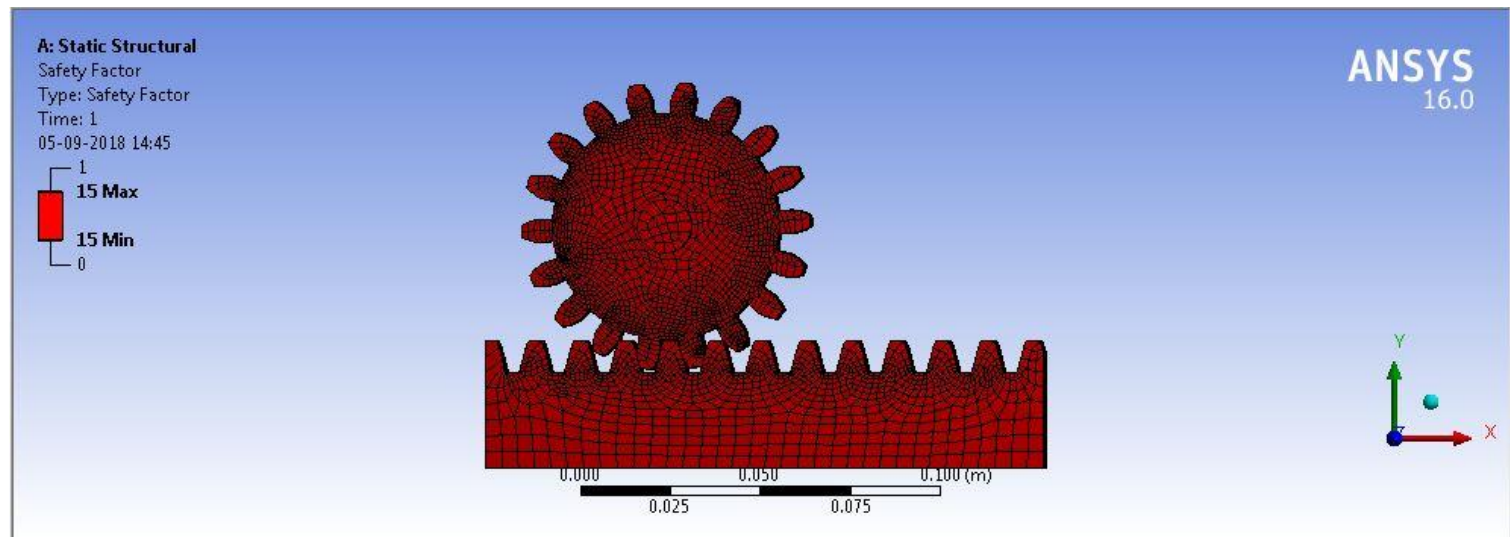

Fig. 11. Factor of safety.

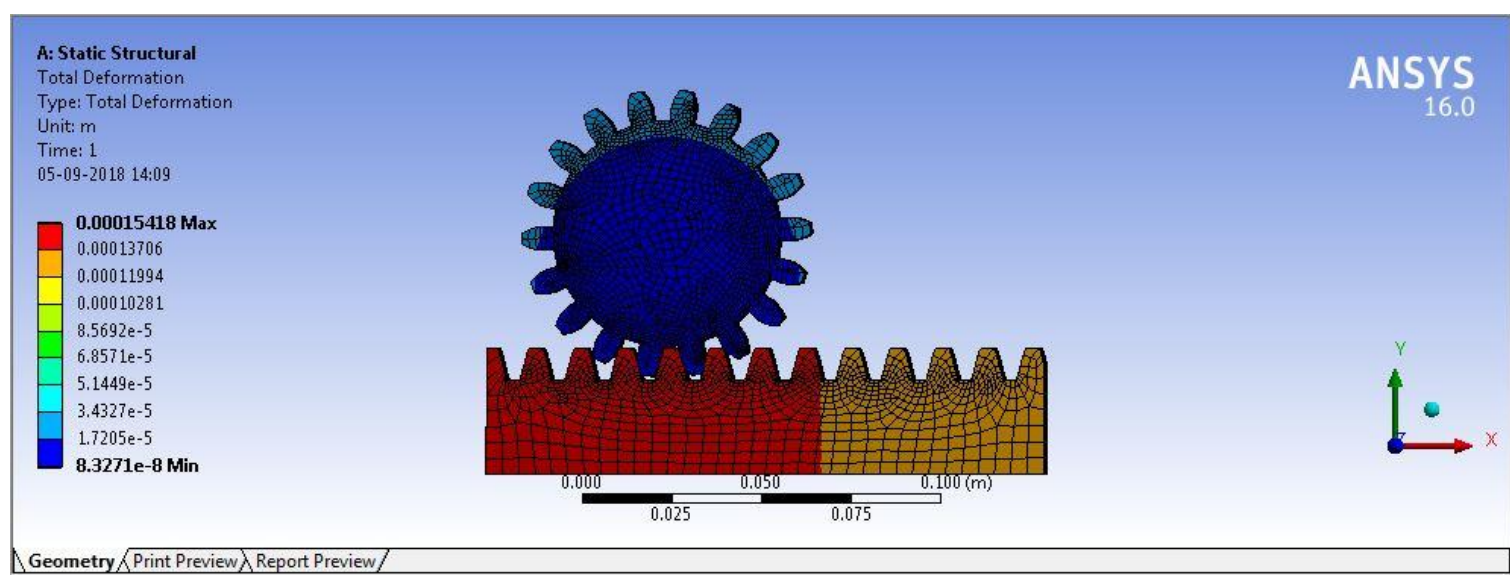

Fig. 12. Total deformation of rack and pinion at $2500 \mathrm{~N}$ using SS.

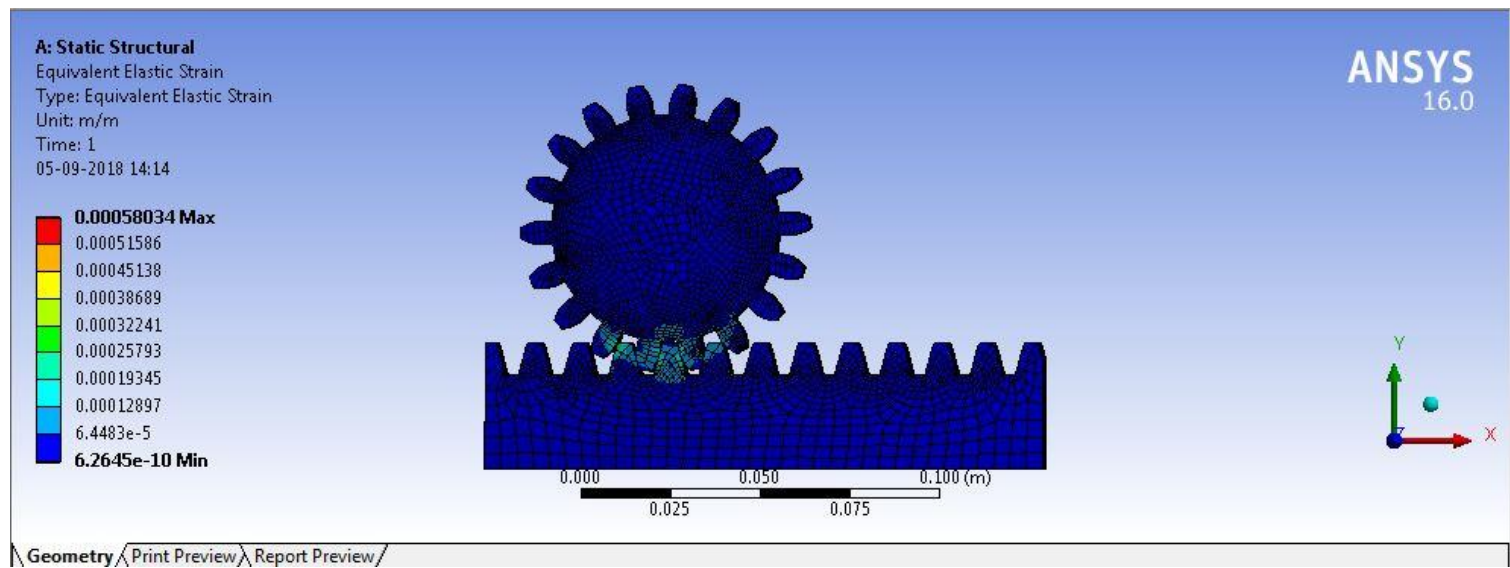

Fig. 13. Equivalent elastic strain of rack and pinion at $2500 \mathrm{~N}$ using SS.

The strain is in the minimum zone except for some strain at the contact area of rack and pinion teeth. The strain is also very low with the value below $2.3214 \times 10^{-5} \mathrm{~m} / \mathrm{m}$ throughout the component. The maximum factor of safety is 15 (Figure 11).

The deformation and strain experienced are higher (i) as the force acting is more in press as shown in Figures 12 and 13. The maximum factor of safety is 15 as shown in Figure 14.

The below analysis states that the maximum deformation occurs at the rack where the force is acting upon and also on the surface contact area of the teeth of rack and pinion. The deformation is high with 
99,496 $\mathrm{m}$ being the maximum. The deformation experienced in PLA is higher than the structural steel material as shown in Figures 15 and 16.

The strain is also very high with the value being below $1.1476 \times 10^{5} \mathrm{~m} / \mathrm{m}$ throughout the component geometry.
The below analysis states that the maximum deformation occurs at the rack where the force is acting upon and also on the surface contact area of the teeth of rack and pinion. The deformation is also very high with $3.3165 \times 10^{5} \mathrm{~m}$ being the maximum. The deformation experienced in PLA is higher than the structural steel material in Figure 17 and Table 1(a,b).

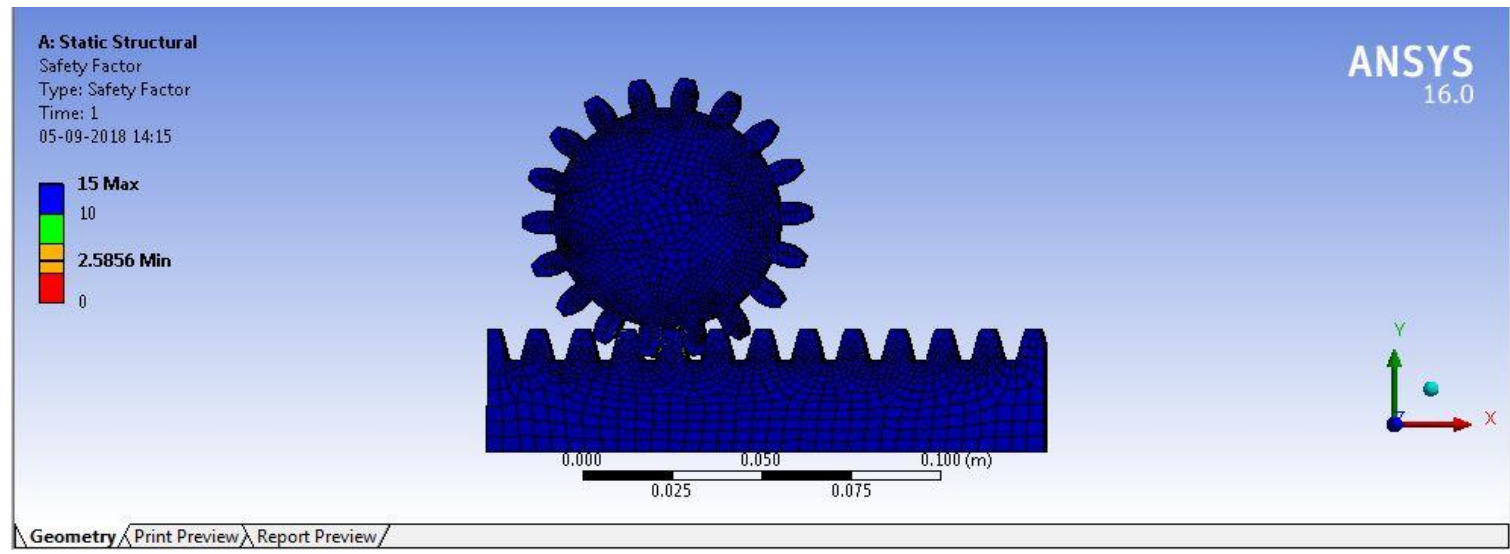

Fig. 14. Factor of safety.

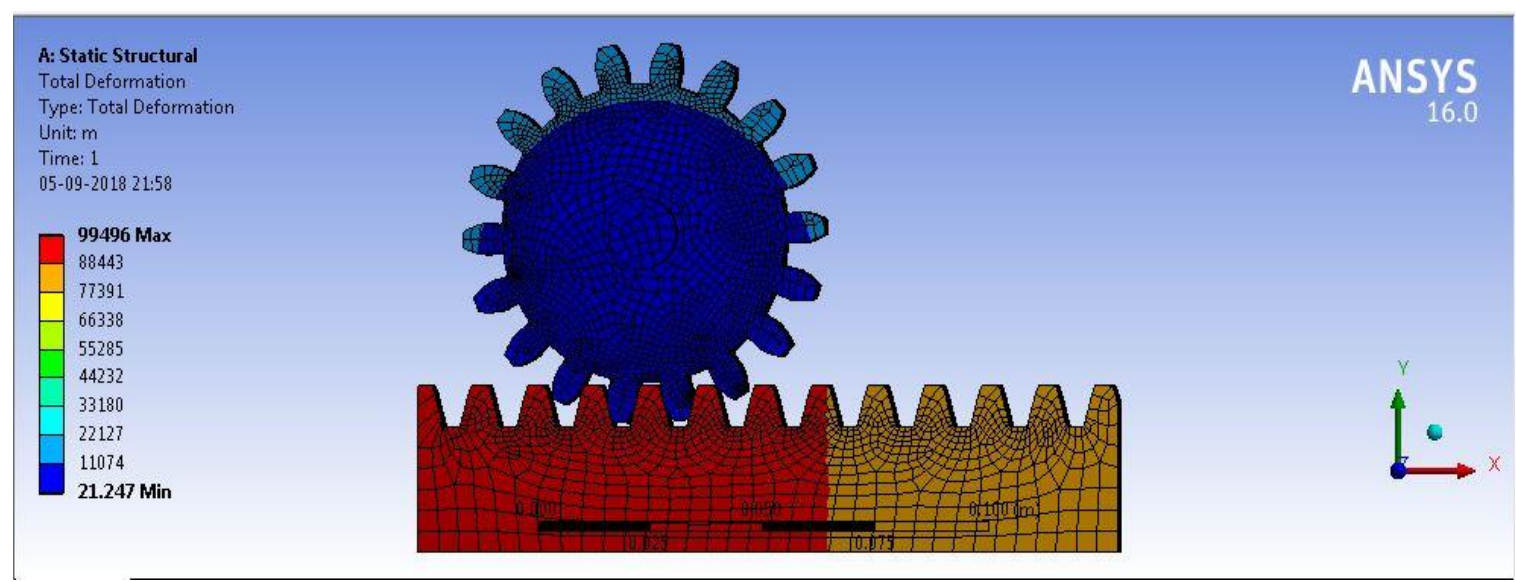

Fig. 15. Total deformation of rack and pinion at $300 \mathrm{~N}$ using PLA.

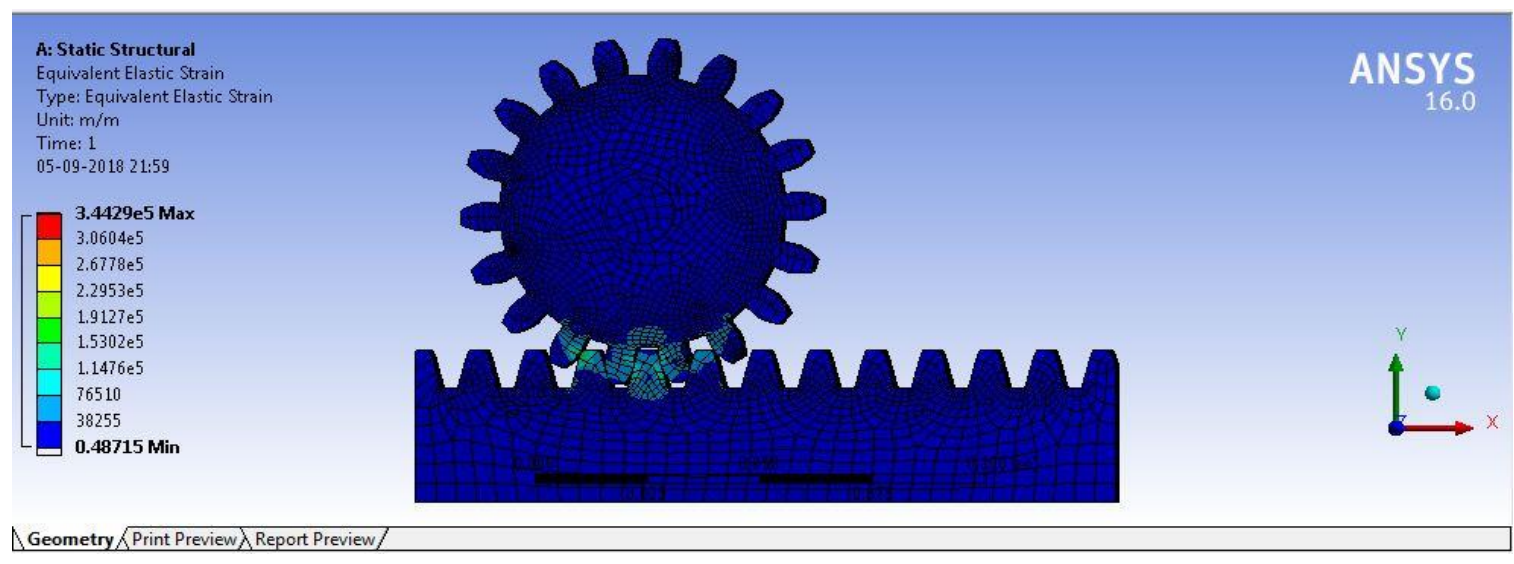

Fig. 16(a). Equivalent elastic strain of rack and pinion at $300 \mathrm{~N}$ using PLA. 


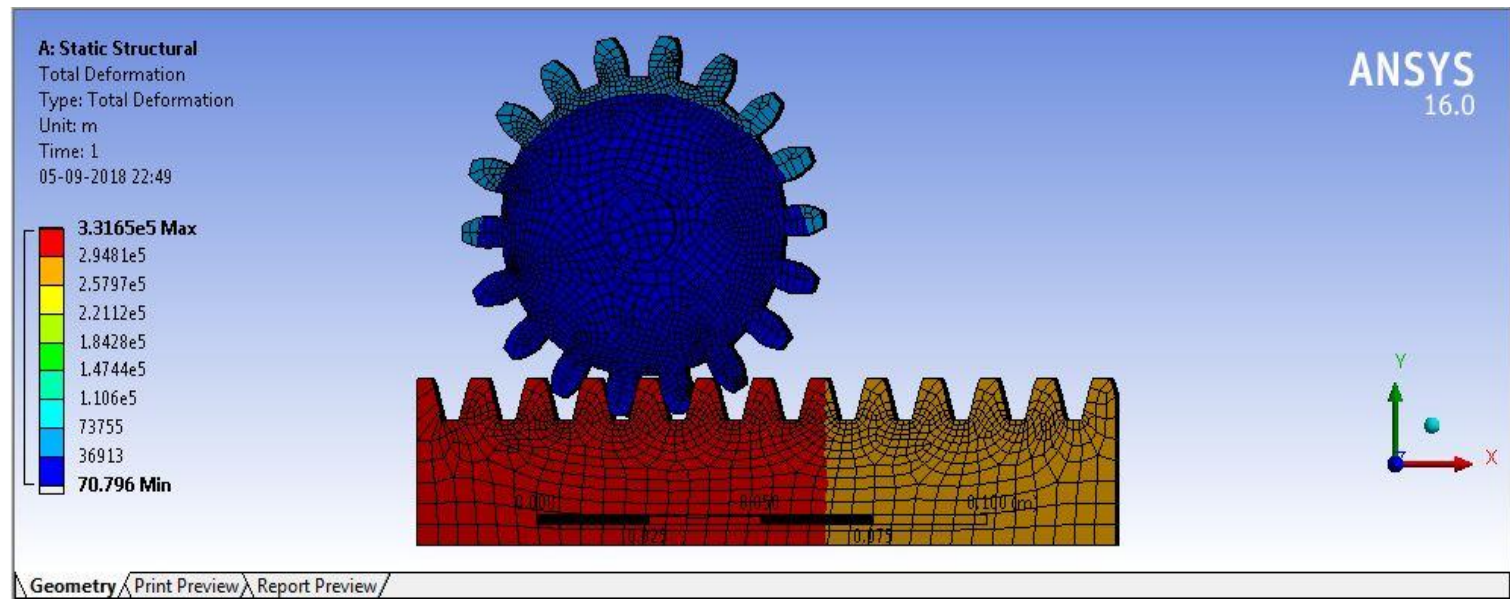

Fig. 16(b). Total deformation of rack and pinion at $1000 \mathrm{~N}$ using PLA.

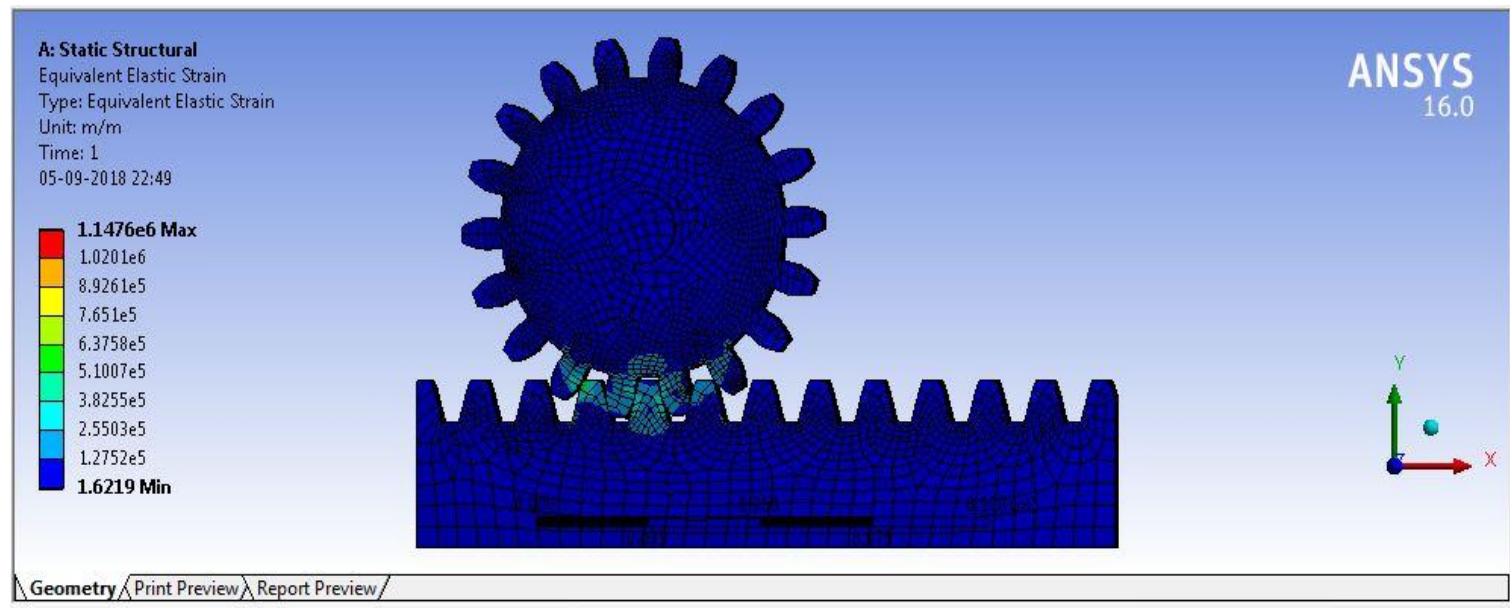

Fig. 17. Equivalent elastic strain of rack and pinion at $1000 \mathrm{~N}$ using PLA.

The strain is also very high with the value being below $3.8225 \times 10^{5} \mathrm{~m} / \mathrm{m}$ throughout the component geometry.

Table 1. Structural analysis results (structural steel).

\begin{tabular}{|c|c|c|c|c|}
\hline Structural steel & $\begin{array}{c}\mathbf{3 0 0} \mathbf{N} \\
\text { Minimum }\end{array}$ & $\begin{array}{c}\mathbf{3 0 0} \mathbf{N} \\
\text { Maximum }\end{array}$ & $\begin{array}{c}\mathbf{2 5 0 0} \mathbf{~ N} \\
\text { Minimum }\end{array}$ & $\begin{array}{c}\mathbf{2 5 0 0} \mathbf{N} \\
\text { Maximum }\end{array}$ \\
\hline $\begin{array}{c}\text { Total deformation } \\
(\mathrm{m})\end{array}$ & $9.9925 \mathrm{e}-9$ & $1.8509 \mathrm{e}-5$ & $7.5245 \mathrm{e}-11$ & $6.9641 \mathrm{e}-5$ \\
\hline $\begin{array}{c}\text { Eq. elastic strain } \\
(\mathrm{m} / \mathrm{m})\end{array}$ & $8.3271 \mathrm{e}-8$ & 0.00015418 & $6.2645 \mathrm{e}-10$ & 0.00058034 \\
\hline
\end{tabular}

Table 1b. Structural analysis results (PLA).

\begin{tabular}{|c|c|c|c|c|}
\hline PLA & $\begin{array}{c}\mathbf{3 0 0} \mathbf{N} \\
\text { Minimum }\end{array}$ & $\begin{array}{c}\mathbf{3 0 0} \mathbf{N} \\
\text { Maximum }\end{array}$ & $\begin{array}{c}\mathbf{1 0 0 0} \mathbf{N} \\
\text { Minimum }\end{array}$ & $\begin{array}{c}\mathbf{1 0 0 0} \mathbf{~ N} \\
\text { Maximum }\end{array}$ \\
\hline $\begin{array}{c}\text { Total deformation } \\
(\mathrm{m})\end{array}$ & 21.247 & 99,496 & 70.796 & $3.3165 \mathrm{e}+5$ \\
\hline $\begin{array}{c}\text { Eq. elastic strain } \\
(\mathrm{m} / \mathrm{m})\end{array}$ & 0.48715 & $3.4429 \mathrm{e}+5$ & 1.6219 & $1.1467 \mathrm{e}+6$ \\
\hline
\end{tabular}

Cura software simulation: Cura software simulation, 3D printed rack and pinion with PLA (scaled down), and PLA scaled-down 3D printed model are shown in Figures 18-20. 


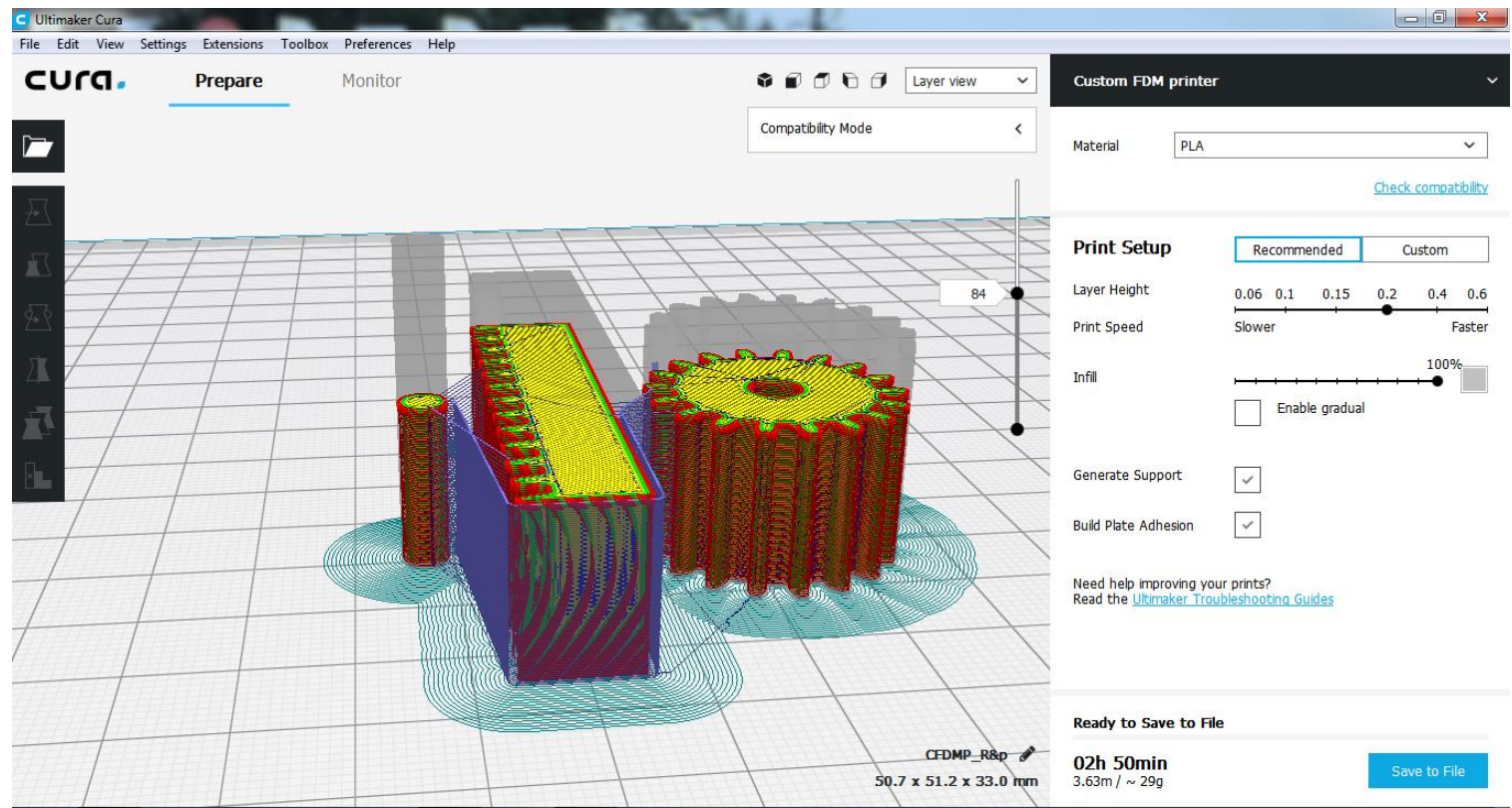

Fig. 18. Cura software simulation.

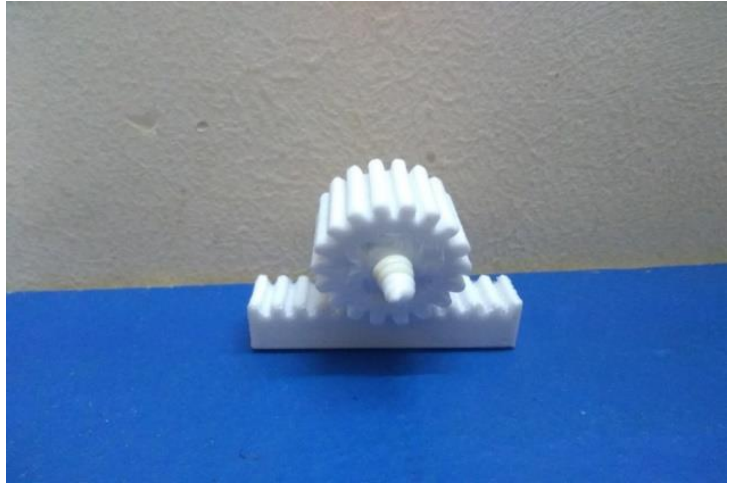

Fig. 19. $3 D$ printed rack and pinion with PLA (scaled down).

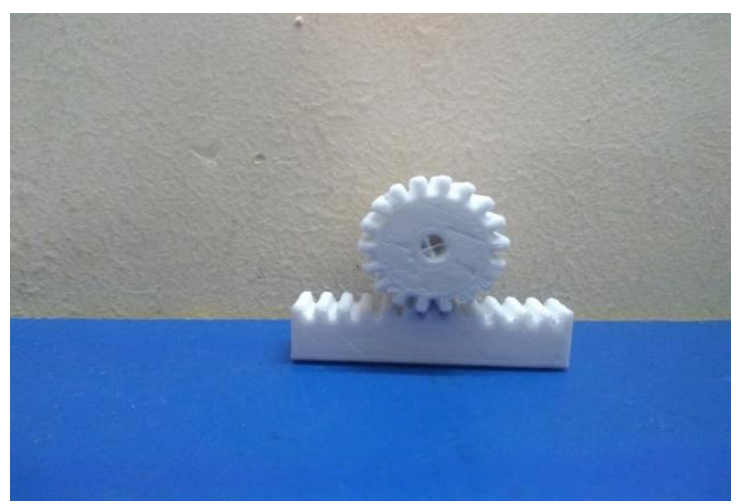

Fig. 20. PLA scaled-down $3 D$ printed model.

\section{CONCLUSION}

Comparing the rack and pinion analysis used in automobile steering, the SS one does have less deformation and strain but is quite heavy. PLA does have a considerably higher deformation but can substitute SS in automobile steering as 300 $\mathrm{N}$ is the maximum parameter taken, and the benefits of using PLA include weight reduction and also reduction in vibration and noise. Thus using a 3D printed rack and pinion steering is beneficial. Comparing the rack and pinion analysis used in press application, the PLA version has a lot of deformation and strain even when a lesser force than SS version is applied. Even SS has more weight, using that is recommended rather than $3 \mathrm{D}$ printed PLA for press application to be on a safer side as the force acting is very high.

\section{REFERENCES}

[1] Chopanea A, Guptab S, Ajitc A, Kakrood S, Salvee A. Design and analysis of plastic gears in rack and pinion steering system for formula supra car. Mat Tod Proc. 2017; 5(2): 5154-5164p.

[2] Hanzaki AR, Rao PVM, Saha SK. Kinematic and sensitivity analysis and optimization of planar rack-and-pinion steering linkages. Mech Mach Theory. 2008; 44(1): 42-56p. 
[3] dos Santosa SP, Brandãob LC, Gallicchioc LH, de Castro Silveiraa Z. Finishing process analysis between honing and hard hobbing in pinion gears applied to a steering system. Energy Proc. 2011; 14: 2-8p.
[4] https://www.chegg.com/homeworkhelp/arbor-press-works-rack-pinionused-develop-large-forces-requchapter-7-problem-2p-solution9780471739326. 